\title{
BMJ Open Pain exposure physical therapy (PEPT) compared to conventional treatment in complex regional pain syndrome type 1 : a randomised controlled trial
}

Karlijn J Barnhoorn, ${ }^{1}$ Henk van de Meent, ${ }^{2}$ Robert T M van Dongen, ${ }^{3}$ Frank P Klomp, ${ }^{4}$ Hans Groenewoud, ${ }^{5}$ Han Samwel, ${ }^{6}$ Maria W G Nijhuis-van der Sanden, ${ }^{1,2}$ Jan Paul M Frölke, ${ }^{7}$ J Bart Staal ${ }^{1,8}$

To cite: Barnhoorn KJ, van de Meent $\mathrm{H}$, van Dongen RTM, et al. Pain exposure physical therapy (PEPT) compared to conventional treatment in complex regional pain syndrome type 1 : a randomised controlled trial. BMJ Open 2015;5:e008283. doi:10.1136/bmjopen-2015008283

- Prepublication history for this paper is available online. To view these files please visit the journal online (http://dx.doi.org/10.1136/ bmjopen-2015-008283).

Received 31 March 2015 Revised 2 September 2015 Accepted 15 October 2015

CrossMark

For numbered affiliations see end of article.

Correspondence to Karlijn J Barnhoorn; Karlijn.Barnhoorn@ radboudumc.nl

\section{ABSTRACT}

Objective: To compare the effectiveness of pain exposure physical therapy (PEPT) with conventional treatment in patients with complex regional pain syndrome type 1 (CRPS-1) in a randomised controlled trial with a blinded assessor.

Setting: The study was conducted at a level 1 trauma centre in the Netherlands.

Participants: 56 adult patients with CRPS- 1 participated. Three patients were lost to follow-up. Interventions: Patients received either PEPT in a maximum of five treatment sessions, or conventional treatment following the Dutch multidisciplinary guideline.

Measurements: Outcomes were assessed at baseline and at 3, 6 and 9 months after randomisation. The primary outcome measure was the Impairment level Sum Score-Restricted Version (ISS-RV), consisting of visual analogue scale for pain (VAS-pain), McGill Pain Questionnaire, active range of motion (AROM) and skin temperature. Secondary outcome measures included Pain Disability Index (PDI); muscle strength; Short Form 36 (SF-36); disability of arm, shoulder and hand; Lower Limb Tasks Questionnaire (LLTQ); 10 m walk test; timed up-and-go test (TUG) and EuroQol-5D.

Results: The intention-to-treat analysis showed a clinically relevant decrease in ISS-RV (6.7 points for PEPT and 6.2 points for conventional treatment), but the between-group difference was not significant $(0.96$, $95 \% \mathrm{Cl}-1.56$ to 3.48 ). Participants allocated to PEPT experienced a greater improvement in AROM (betweengroup difference $0.51,95 \% \mathrm{Cl} 0.07$ to $0.94 ; \mathrm{p}=0.02$ ). The per protocol analysis showed larger and significant between-group effects on ISS-RV, VAS-pain, AROM, PDI, SF-36, LLTQ and TUG.

Conclusions: We cannot conclude that PEPT is superior to conventional treatment for patients with CRPS-1. Further high-quality research on the effects of PEPT is warranted given the potential effects as indicated by the per protocol analysis.

Trial registration numbers: NCT00817128 and NTR 2090.

\section{Strengths and limitations of this study}

- The trial was prospectively registered and followed published protocol.

- Patients were randomised, allocation was concealed and the assessor was blinded.

- Low inclusion rate, underpowered trial.

- Substantial number of patients switched.

- Ten patients in the pain exposure physical therapy (PEPT) group received some form of medication or other conventional treatment.

\section{INTRODUCTION}

Complex regional pain syndrome type 1 (CRPS-1) is defined by the International Association for the Study of Pain (IASP) as a pain syndrome that can develop after physical injury and is characterised by pain, in combination with sensory, autonomic, motor and/or trophic changes. The symptoms exceed the expected clinical course of the inciting event. ${ }^{1}$

CRPS-1 is seen as a multifactorial disorder that requires a multidisciplinary approach. The variability in the activation of the three major pathophysiological pathways (aberrant inflammatory mechanisms, vasomotor dysfunction and maladaptive neuroplasticity) involved in CRPS cause the clinical heterogeneity. ${ }^{2}$ Different stages have been identified, ${ }^{2}{ }^{3}$ and over time, clinical features spread and may even occur in the opposite or ipsilateral limb. ${ }^{45}$

To date, there are many uncertainties regarding the most optimal way of managing this disabling disorder, which in many cases appears to be therapy resistant. Recent systematic reviews show that there is still insufficient evidence for the effectiveness of most therapies. $^{6} 7$ The Cochrane systematic review 
from 2013 concluded that there is low-quality evidence for many treatment modalities such as the use of bisphosphonates, calcitonin or a daily course of intravenous ketamine; graded motor imagery; and physical or occupational therapy. The review authors generally identified a critical lack of high-quality evidence for the effectiveness of most therapies. ${ }^{7}$

Despite the lack of high-quality evidence, the Dutch national multidisciplinary clinical guideline for the treatment of CRPS-1 was issued in 2006. ${ }^{8}$ Treatment recommendations in this guideline include pharmacological interventions aimed primarily at decreasing pain combined with physical and/or occupational therapy. This therapy is focused on controlling pain and other symptoms, using mild exposure, and increasing the capacity step by step in a pain-contingent manner. Pharmacological treatment includes the use of analgesics in a step-up procedure in accordance with the WHO pain ladder, free radical scavengers including dimethyl sulfoxide $50 \%$ ointment (DMSO) and $\mathrm{N}$-acetylcysteine, calcium channel blockers and ketanserin. Patients presenting with allodynia or hyperalgesia are given gabapentin, amitriptyline or carbamazepine. Dystonia, myoclonia and muscle spasms are treated with baclofen, diazepam or clonazepam. In the case of cold skin, the anaesthesiologist will prescribe vasodilating drugs such as verapamil, ketensin and pentoxifylline. In case of insufficient clinical effect, sympathetic blockade or spinal cord stimulation can be considered. ${ }^{8}$

In contrast to the biomedical approach as advocated in the guideline, the attention of the research community has shifted over past decades towards a behavioural paradigm for the management of benign chronic pain disorders. A widely used model in treatment of patients with chronic pain is the fear-avoidance model of Vlaeyen. ${ }^{9}$ When injury leads to pain, rest and avoidance of movement may be adaptive in the acute phase, but in the longer term, this 'protective' responding is no longer functional and may paradoxically worsen the problem. ${ }^{10}{ }^{11}$ Decreased motor activity can lead to changes in cortical representation zones, ${ }^{12}$ which have been identified in patients with CRPS-1. ${ }^{13}$ Movements are more effortful, and unsuccessful motor attempts lead to compensatory behaviour. Unpleasant and unsuccessful experiences, in combination with pain increase, lead to increased pain-avoidance behaviour, which causes so-called learnt non-use. ${ }^{12}$

In order to overcome the hypothesised learnt non-use, some treatment approaches apply a well-known and accepted strategy in psychology: graded exposure to fearful stimuli in order to overcome fear and avoidance behaviour. Patients with CRPS-1 are instructed to use their affected limb step by step, using positive reinforcement and under explanation that motor activities are not harmful but rather enhance recovery.

A more direct alternative is pain exposure physical therapy (PEPT, table 1). ${ }^{14}{ }^{15}$ PEPT is not similar to graded exposure, as PEPT is neither limited to patients with high levels of fear nor uses a strict hierarchy of avoided activities to which patients need to be exposed gradually, as is typical for graded exposure. In PEPT, patients are directly exposed to painful stimuli (ie, activities). They are stressed to regain their normal daily

Table 1 Description of interventions according to the TIDieR checklist

\begin{tabular}{|c|c|c|}
\hline & Pain exposure physical therapy & Conventional treatment \\
\hline \multirow[t]{2}{*}{ Why } & Pain is a false warning sign & Pain is a sign of dysfunction \\
\hline & $\begin{array}{l}\text { Rapidly regaining functional activity, despite levels of } \\
\text { pain }\end{array}$ & $\begin{array}{l}\text { Pain contingent, improving functional activity while } \\
\text { controlling pain }\end{array}$ \\
\hline \multirow[t]{6}{*}{ What } & Exposure to painful movements and activities; & Dependent of pain limits; \\
\hline & $\begin{array}{l}\text { No medication, no TENS, no walking aids, splints or } \\
\text { bandages; }\end{array}$ & $\begin{array}{l}\text { Medication (analgesics, free radical scavengers, } \mathrm{Ca}^{2+} \\
\text { channel blockers, etc), TENS, walking aids, splints, }\end{array}$ \\
\hline & Self-massage, 'forced' use, progressive-loading & bandages; \\
\hline & $\begin{array}{l}\text { exercises, muscle strength training, joint mobility } \\
\text { exercises; }\end{array}$ & $\begin{array}{l}\text { Mild exposure, progressive-loading exercises, muscle } \\
\text { strength training, joint mobility exercises; }\end{array}$ \\
\hline & $\begin{array}{l}\text { Information and education about CRPS-1, PEPT } \\
\text { and the role of chronic pain as a false warning sign; } \\
\text { Internal locus of control }\end{array}$ & $\begin{array}{l}\text { Information and education about CRPS- } 1 \text { and the role } \\
\text { of pain as a protective response and a sign of } \\
\text { dysfunction; }\end{array}$ \\
\hline & & External locus of control \\
\hline Who & Two physical therapists; & Anaesthesiologist, physical therapist, rehabilitation \\
\hline provided & Partner as a 'home coach' & physician \\
\hline How & $\begin{array}{l}\text { Face-to-face, individually, explicit home exercises } \\
\text { and functioning in activities of daily living }\end{array}$ & Face-to-face, individually \\
\hline Where & PT department, continuously in daily life & Anaesthesiology department, PT department \\
\hline How much & Maximum five sessions of $40 \mathrm{~min}$ & No predefined limits, on average $15-20$ sessions \\
\hline Tailoring & $\begin{array}{l}\text { Adapted to individual competencies and daily life } \\
\text { requirements }\end{array}$ & Adapted to levels of pain \\
\hline
\end{tabular}


activities as soon as possible, without the use of medication, and are instructed to ignore their pain. It is explained to them that pain in itself has become a 'false warning sign' due to long-lasting functional disturbances in the affected limb. On the basis of the history taking and observations during assessment, the intervention is adapted to the individual patient. During the first assessment, patients, along with their partners, set their personal goals for treatment. They receive detailed information about the condition, the working mechanism of pain and pain-related avoidance behaviour, and the rationale and content of PEPT. The therapists inform the patients that, although understanding the pain, they will not respond to it: they will ignore both verbal and non-verbal expressions of pain during treatment. Because of improved function, the pain will eventually diminish. All medication aimed at CRPS-1, including analgesics, is stopped and use of crutches and other walking aids, splints and bandages is discouraged. Patients learn how to decrease skin sensitivity for touch and pressure by performing self-massage and 'forced' use of the affected extremity in daily activities. The tailored progressive-loading exercises focus on specific daily activities, using muscle strength training and joint mobility exercises, both passive and active. The patients perform the exercises at home and incorporate them in their daily life using regular daily schedules. The physical therapists act mainly as instructors and coaches, and confirm and reward progression with positive feedback. They instruct the partners to change their protective and curative role into a facilitating, motivating and coaching role, removing barriers without taking over. The partners have to ignore the pain behaviour of the patients during the treatment sessions and home exercises. The key to success of PEPT is internal motivation and adherence. ${ }^{14}$

It has already been shown that PEPT is a promising treatment for patients with CRPS- $1 .{ }^{14} 15$

\section{Objective}

The objective of the current study is to evaluate the effectiveness of this novel CRPS-1 treatment with PEPT compared with the currently best available care in the Netherlands, which is treatment according to the Dutch multidisciplinary guideline for CRPS- $1 .{ }^{8}$

In accordance with the TIDieR (Template for Intervention Description and Replication) checklist, ${ }^{16}$ descriptions of both interventions under study can be found in table 1 .

\section{METHODS}

\section{Design overview}

We designed this trial as a randomised controlled trial with blinding of the assessor. The assessor (TT) conducted the measurements at baseline and at 3,6 and 9 months follow-up. The experimental and control interventions were performed by two different teams. The study protocol and rationale of both treatment strategies have been published elsewhere. ${ }^{17}$

We applied the consolidated standards of reporting trials (CONSORT) guidelines. This trial was prospectively registered at http://www.clinicaltrials.gov, NCT0081 7128, and at http://www.trialregister.nl, NTR 2090.

\section{Setting and participants}

The trial was conducted in a university hospital, a level 1 trauma centre in the Netherlands. Approximately 150 new adult patients visit our specialised clinic each year, of whom $23 \%$ are diagnosed with CRPS- $1 .{ }^{18}$ This means that in our clinic 30-40 patients are diagnosed with CRPS-1 each year. To increase the amount of eligible patients for our trial, we approached hospitals and general practitioners in our region to help recruit potential participants. ${ }^{17}$ A rehabilitation physician (HvdM) and a surgeon (JPMF) performed physical examination to determine whether patients were eligible and the diagnosis of CRPS-1 could be confirmed using the research diagnostic criteria for CRPS as proposed by Harden et al. ${ }^{19}$

Diagnostic CRPS criteria:

1. Continuing pain, which is disproportionate to any inciting event

2. At least one symptom in each of the four following categories:

- Sensory: reports of hyperalgesia and/or allodynia

- Vasomotor: reports of temperature asymmetry, skin colour changes and/or skin colour asymmetry

- Sudomotor/oedema: reports of oedema, sweating changes and/or sweating asymmetry

- Motor/trophic: reports of decreased range of motion, motor dysfunction and/or trophic changes

3. At least one sign in two or more of the following categories:

- Sensory: evidence of hyperalgesia and/or allodynia

- Vasomotor: evidence of temperature asymmetry $\left(>1^{\circ} \mathrm{C}\right)$, skin colour changes and/or skin colour asymmetry

- Sudomotor/oedema: evidence of oedema, sweating changes and/or sweating asymmetry

- Motor/trophic: evidence of decreased range of motion, motor dysfunction and/or trophic changes

4. There is no other diagnosis that better explains the signs and symptoms.

Other inclusion criteria were that the first assessment took place between 3 and 24 months after the inciting event and patients were between 18 and 80 years of age. In case of other causes for the signs and symptoms (eg, non-union, osteomyelitis and CPRS-2), patients were excluded (JPMF and HvdM). The exclusion criteria included CRPS-1 in more than one extremity, relapse of CRPS-1, pregnancy, lactation and prior sympathectomy of the affected extremity.

The trial lasted from 9 January 2009 to 7 March 2012. 


\section{Blinding}

The trained research nurse (TT) who performed all assessments prior to randomisation and during follow-up was blinded to the treatment allocation. Given the nature of the interventions, it was not possible to blind the patients and the therapists, although both treatment teams did not have contact with each other. Patients were instructed not to discuss their treatment with the research nurse.

\section{Randomisation and interventions}

A statistician who had no clinical involvement in the trial (HG) designed a computerised randomisation program. An independent person (MR) allocated patients to one of the two treatment groups (PEPT or conventional treatment $(\mathrm{CONV})$, table 1$)$ in a 1:1 ratio using the randomisation program. The researcher (JPMF) who determined the eligibility of patients was unaware of the randomisation sequence.

\section{Pain exposure physical therapy}

PEPT was conducted by two physical therapists together (FPK and AP, FPK and MT), who were trained by a psychologist to actively interact with the patient and his or her partner during the treatment sessions. PEPT consisted of a maximum of five treatment sessions, each of 40 min duration, with varying intervals between the sessions depending on the progression and personal needs of the patient. Treatment was ceased when the patients met their predefined functional treatment goals, or when they showed insufficient motivation and selfefficacy, strong resistance or insufficient results. ${ }^{14} 1517$

\section{Conventional treatment}

A different group of physicians carried out CONV. This treatment included pharmacological interventions (RTMvD) combined with physical therapy in a more pain-contingent manner (PPM, JO and RTMvD). ${ }^{8}$ The physician carrying out the pharmacological intervention was based at a remote location of our university hospital.

\section{Treatment compliance and adverse events}

We measured treatment compliance by documenting the attendance to physical therapy (either PEPT or $\mathrm{CONV}$ ) and to the physician, and reporting use of medication. During each visit, abidance to treatment was checked. Side effects were documented in the patient's medical record. We used standardised serious adverse event forms for reporting (serious) adverse events.

\section{Outcomes}

The primary outcome measure was the Impairment level Sum Score-Restricted Version (ISS-RV), which consists of three measurement parameters focusing on typical signs and symptoms of CRPS-1 (pain, active range of motion (AROM) and temperature). ISS-RV contains four measurement instruments: visual analogue scale for pain (VAS-pain), ${ }^{20}$ McGill Pain Questionnaire Dutch Language Version (MPQ-DLV), ${ }^{21}$ AROM of joints ${ }^{22}$ and skin temperature. ${ }^{23}$ Each element of ISS-RV is converted to a 1-10 points scale, which results in a sum score ranging from 4 to 40 points. ${ }^{24-26} \mathrm{~A}$ higher value refers to a more severe impairment. We consider a difference of four ISS-RV points as clinically relevant. ${ }^{27}$

We based the secondary outcome measures on the WHO International Classification of Functioning, Disability and Health. ${ }^{28}$ We included:

1. The Pain Disability Index ${ }^{29}$ (PDI) to measure disability and the level of activities;

2. Muscle strength ${ }^{30}$ to measure functioning (grip strength for the upper extremity and strength of dorsiflexors and plantar flexors of the ankle for the lower extremity);

3. Short Form 36 (SF-36) Questionnaire ${ }^{31}$ to measure the level of participation;

4. Disability of the arm, shoulder and hand, DLV (DASH-DLV) questionnaire ${ }^{32}$ to measure activities involving the upper extremity;

5. Lower Limb Tasks Questionnaire (LLTQ) $;^{33}$

6. 10-metre walk test (10MWT) $;^{34-36}$

7. Timed up-and-go test (TUG) ${ }^{36-39}$ to measure activities related to the lower extremity;

8. Quality of life, using the EuroQol-5D (EQ-5D) index and VAS. ${ }^{40}$

We also asked patients how well they could perform the physical therapy.

The cost-effectiveness analysis and analysis of psychological outcome measures (eg, Pain Catastrophising Scale) are ongoing and we will present them in subsequent publications.

\section{Sample size calculation}

The sample size calculation was based on an expected proportion of responders of $80 \%$ in the PEPT group ${ }^{14}$ and $50 \%$ in the CONV group. ${ }^{27}$ Given an $\alpha$ of 0.05 and a power of $80 \%$ for a one-sided $\chi^{2}$ test, 62 patients had to be included when using a 1:1 randomisation.

\section{Statistical analysis}

$\mathrm{KJB}$ and $\mathrm{HG}$ assembled all measured data in a database and analysed them using SPSS V.20.0 for Windows (SPSS Inc, Chicago, Illinois, USA). Baseline characteristics of the patients and baseline values of the outcome measures were described using measures of central tendency and dispersion. We conducted the statistical analysis following the intention-to-treat principle (ITT): patients were analysed in the groups to which they were allocated. We also performed a secondary analysis following the per protocol (PP) principle: only the patients who followed the randomisation protocol were analysed and the patients who opted out of the treatment allocation prior to treatment initiation were excluded from this analysis. We performed a responder analysis in order to compare the proportion of patients achieving the minimal clinically important difference (MCID) of four 
points on ISS-RV. We used linear mixed models with unstructured repeated covariance to determine the between-group differences over time. Treatment, measurement in time, and treatment $\times$ time interaction were added to the model as factors and outcome at baseline was added as a covariate. Linear mixed models deal with missing data by predicting the best-fitting line for each patient without data imputation.

\section{Role of the funding source}

The Netherlands Organisation for Health Research and Development (ZonMw; 1709901004) sponsored this study. The sponsor had no role in the study design, data collection, data analysis, data interpretation or writing of the report.

\section{RESULTS}

\section{Study participants}

Between January 2009 and June 2011, 432 patients were referred to the CRPS outpatient department of our hospital. Of these patients, 328 (75.9\%) were CRPS negative and another 30 did not meet the remaining inclusion criteria. Seventy-four patients fulfilled the study inclusion criteria and were invited to participate in the study. Eighteen patients did not give their informed consent due to constraints like travel time or an unwillingness to undergo frequent assessments. The resulting 56 patients were randomly allocated to either PEPT $(n=28)$ or CONV ( $n=28)$. Prior to treatment initiation, four patients (14\%) in the PEPT group and 11 patients $(39 \%)$ in the CONV group opted out of their assigned treatment and switched groups.
Figure 1 shows the participant flow. During the course of the trial, three additional patients switched from CONV to PEPT due to insufficient results with their treatment.

One patient in the PEPT group dropped out after two treatment sessions because of complete recovery. One patient in the CONV group was not confident about the treatment and was lost to follow-up. Another patient in the CONV group stopped without offering a reason.

Table 2 shows the baseline characteristics of the groups. The baseline values of the primary and secondary outcome measures are shown in the first columns of tables 3 and 4, respectively.

Six patients in the PEPT group received analgesics from their general practitioner, but it was unclear whether this was CRPS related. One patient received DMSO and amitriptyline $10 \mathrm{mg}$, one patient received Arthrotec (diclofenac $50 \mathrm{mg} / \mathrm{misoprostol} 200 \mu \mathrm{g}$ and diclofenac $75 \mathrm{mg} /$ misoprostol $200 \mu \mathrm{g}$ ), one received paracetamol/codeine phosphate $(500 / 10 \mathrm{mg})$, one received ibuprofen $(600 \mathrm{mg})$ and omeprazol $(20 \mathrm{mg})$, one received diclofenac $(50 \mathrm{mg})$ for 1 month and one patient received Zaldiar (tramadol $37.5 \mathrm{mg} /$ paracetamol $325 \mathrm{mg}$ ) for 10 days. Two patients in the PEPT group received some form of CONV. Two patients who were allocated to the CONV group and switched to PEPT prior to treatment initiation also received some form of CONV; one patient received DMSO, oxynorm, ondansetron, oxycontin, lyrica and primperan; one patient received diclofenac, transcutaneous electrical nerve stimulation and standard physical therapy.

All other patients received their treatment according to the methods described in the intervention section. None of the patients received spinal cord stimulation or sympathetic blockade.
Figure 1 Trial profile (CONV, conventional treatment; ITT, intention-to-treat; PP, per protocol; PEPT, pain exposure physical therapy).

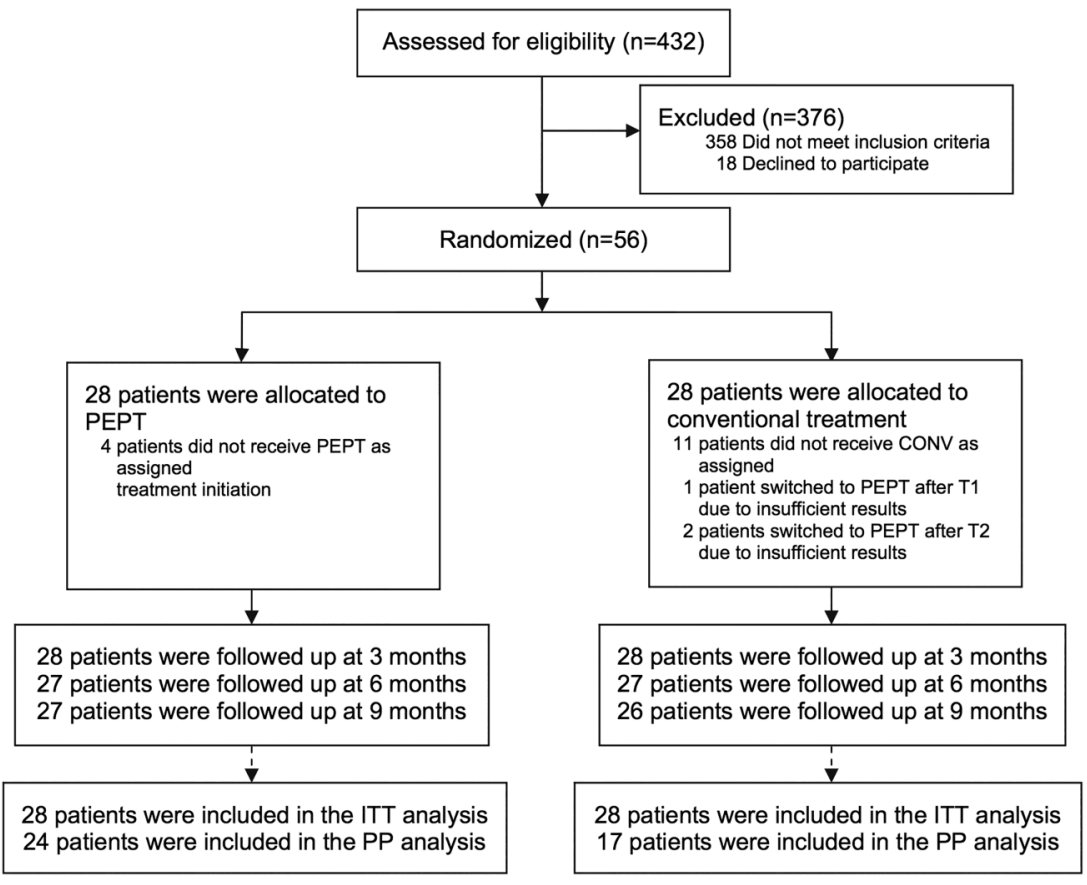


Table 2 Baseline characteristics of participants

\begin{tabular}{|c|c|c|c|c|c|}
\hline \multirow[b]{2}{*}{ Variable } & \multirow{2}{*}{$\begin{array}{l}\text { Overall } \\
(n=56)\end{array}$} & \multicolumn{2}{|c|}{ Intention-to-treat } & \multicolumn{2}{|l|}{ Per protocol } \\
\hline & & PEPT $(n=28)$ & CONV $(n=28)$ & PEPT $(n=24)$ & CONV $(n=17)$ \\
\hline Age $\left(\right.$ years) ${ }^{\star}$ & $44.3(16.6)$ & $43.7(14.8)$ & $44.9(18.5)$ & $43.1(15.6)$ & 46.1 (17.9) \\
\hline Women $(n)$ & 45 (80.4\%) & $24(85.7 \%)$ & $21(75.0 \%)$ & $20(83.3 \%)$ & $12(70.6 \%)$ \\
\hline Upper extremity $(n)$ & $37(66.1 \%)$ & $18(64.3 \%)$ & $19(67.9 \%)$ & $15(62.5 \%)$ & $14(82.4 \%)$ \\
\hline Dominant side affected (n) & $32(57.1 \%)$ & $13(46.4 \%)$ & $19(67.9 \%)$ & $12(50.0 \%)$ & $14(82.4 \%)$ \\
\hline Time since event (months) ${ }^{*}$ & $7.2(4.1)$ & $7.0(3.8)$ & $7.5(4.5)$ & $7.0(4.0)$ & $8.1(4.9)$ \\
\hline
\end{tabular}

*Mean (SD)

CONV, conventional treatment; PEPT, pain exposure physical therapy.

\section{Outcomes}

Table 3 shows the values at all measurement points of ISS-RV and its constituents. The values of the secondary outcome measures are shown in table 4 . Table 5 displays the estimated group differences for both the ITT analysis and the PP analysis. The factor treatment $\times$ time interaction was not significant, and we therefore left it out of the linear mixed models.

In the PEPT group, $63 \%$ of the patients achieved the MCID, compared to $56 \%$ in the CONV group, with a risk ratio of $1.12(95 \%$ CI 0.72 to 1.77$)$. A responder analysis showed no significant association between treatment and achieving the MCID: $\chi^{2}$ (1) $=0.26, \mathrm{p}=0.41$.

The ITT analysis showed a decrease in ISS-RV of 6.7 points for the PEPT group and 6.2 points for the CONV group (estimated group difference $0.96,95 \%$ CI -1.56 to 3.48; $\mathrm{p}=0.45$ ) over 9 months (figure 2). The AROM showed a significant difference between groups with a decrease of 1.8 points in the PEPT group and 1.6 points in the CONV group $(0.51,95 \%$ CI 0.07 to $0.94 ; \mathrm{p}=0.02)$. VAS-pain, MPQ and skin temperature, as well as the secondary outcome measures, showed improvement over time, but there were no significant between-group differences in the ITT analysis.

The PP analysis showed greater improvement and differences between treatment groups in favour of PEPT, as shown in table 5 . The PP estimated between-group differences in ISS-RV was $2.90 \quad(95 \%$ CI 0.10 to 5.70 ; $\mathrm{p}=0.04)$, VAS-pain decreased 1.61 points more in the PEPT group than in the CONV group (95\% CI 0.06 to 3.16; $\mathrm{p}=0.04$ ) and the difference in AROM between the groups was 0.75 points (95\% CI 0.28 to 1.23 ; $p=0.003$ ). According to the PP analysis, patients in the PEPT group also had a significantly larger improvement in PDI, SF-36, LLTQ and TUG. The other outcome measures improved over time for both treatment groups, but between-group differences were not significant.

Most of the treatment effects were reached at 3 months after inclusion and remained over time.

Patients in the PEPT group had a mean of four treatment sessions, whereas patients in the CONV group visited their physical therapists 17 times on average. The physical therapy was feasible for $75.0 \%$ of the patients in the PEPT group compared to $72.7 \%$ of patients in the CONV group.

Table 3 Descriptives of the primary outcome measures for the intention-to-treat analysis

\begin{tabular}{|c|c|c|c|c|}
\hline Variable & Baseline & 3 months & 6 months & 9 months \\
\hline \multicolumn{5}{|c|}{ ISS-RV (range 4-40)* } \\
\hline PEPT & $21.00(5.30)$ & $14.94(5.84)$ & $14.86(6.13)$ & $14.30(5.88)$ \\
\hline CONV & $21.12(5.31)$ & $16.43(6.25)$ & $15.03(6.35)$ & $14.92(5.28)$ \\
\hline \multicolumn{5}{|c|}{ VAS-pain (range $1-10)^{\star}$} \\
\hline PEPT & $6.18(2.50)$ & $4.41(2.85)$ & $4.31(2.81)$ & $3.52(2.69)$ \\
\hline CONV & $7.11(2.01)$ & 5.35 (3.09) & $4.92(3.34)$ & $4.96(3.02)$ \\
\hline \multicolumn{5}{|c|}{ McGill Pain Questionnaire (range 1-10)* } \\
\hline PEPT & $5.73(2.11)$ & $4.33(1.97)$ & $3.78(2.30)$ & $3.60(1.70)$ \\
\hline CONV & $5.15(1.43)$ & $4.36(1.91)$ & $3.90(2.05)$ & $3.29(1.88)$ \\
\hline \multicolumn{5}{|c|}{ Active range of motion (range $1-10)^{*}$} \\
\hline PEPT & $4.71(2.16)$ & $3.11(1.26)$ & $3.35(1.67)$ & $2.89(1.22)$ \\
\hline CONV & 4.93 (1.98) & $4.04(1.95)$ & $3.52(1.26)$ & $3.32(0.95)$ \\
\hline \multicolumn{5}{|c|}{ Skin temperature (range $1-10)^{\star}$} \\
\hline PEPT & 4.39 (2.91) & $3.07(2.39)$ & $3.50(2.52)$ & $4.26(3.15)$ \\
\hline CONV & $3.96(3.35)$ & $2.92(2.74)$ & $3.00(2.23)$ & $3.32(2.45)$ \\
\hline
\end{tabular}


Table 4 Descriptives of the secondary outcome measures for the intention-to-treat analysis

\begin{tabular}{|c|c|c|c|c|}
\hline Variable & Baseline & 3 months & 6 months & 9 months \\
\hline \multicolumn{5}{|c|}{ Pain Disability Index (range $0-70)^{\star}$} \\
\hline PEPT & $36.08(11.38)$ & $22.88(14.44)$ & $14.33(14.37)$ & $14.49(14.80)$ \\
\hline CONV & $34.12(14.59)$ & $22.92(15.91)$ & $18.37(14.49)$ & $15.94(15.34)$ \\
\hline \multicolumn{5}{|c|}{ Muscle strength $(\%)^{\star} \dagger$} \\
\hline PEPT & $61.90(22.96)$ & $36.80(27.86)$ & $27.50(26.52)$ & $25.83(27.39)$ \\
\hline CONV & $67.14(23.16)$ & $46.10(26.16)$ & $38.25(27.20)$ & $32.50(27.22)$ \\
\hline \multicolumn{5}{|c|}{ SF-36 (range 0-100)‡ } \\
\hline PEPT & $48.17(15.31)$ & $60.90(17.55)$ & $73.98(13.63)$ & $73.30(17.49)$ \\
\hline CONV & $47.60(16.85)$ & 58.35 (20.73) & $66.39(17.42)$ & $68.57(18.90)$ \\
\hline \multicolumn{5}{|c|}{ DASH (range $0-100)^{\star} \S$} \\
\hline PEPT $(n=18)$ & $57.33(13.54)$ & $37.00(17.70)$ & $28.79(19.88)$ & $28.57(19.88)$ \\
\hline $\operatorname{CONV}(n=19)$ & $58.27(12.18)$ & $43.45(22.91)$ & $35.59(21.19)$ & $27.52(22.00)$ \\
\hline \multicolumn{5}{|c|}{ LLTQ daily activity (range $0-40) \neq \eta^{\star \star}$} \\
\hline PEPT $(n=9)$ & $17.33(8.12)$ & $24.75(4.80)$ & $32.84(5.02)$ & $36.71(3.90)$ \\
\hline CONV $(n=7)$ & $15.14(8.45)$ & $22.00(10.53)$ & $23.70(11.08)$ & 25.79 (11.92) \\
\hline \multicolumn{5}{|l|}{$10 \mathrm{MWT}$ (in s)* } \\
\hline PEPT $(n=10)$ & 35.78 (39.19) & $15.84(2.23)$ & $15.33(4.37)$ & $16.16(8.25)$ \\
\hline CONV $(n=9)$ & $62.86(58.37)$ & $75.50(147.29)$ & $25.22(16.48)$ & $20.17(10.73)$ \\
\hline \multicolumn{5}{|l|}{ TUG (in s) ${ }^{\star} \emptyset$} \\
\hline PEPT $(n=10)$ & $11.78(8.51)$ & $36.03(94.20)$ & $6.12(1.51)$ & $6.47(2.87)$ \\
\hline CONV $(n=9)$ & $22.40(25.04)$ & 22.40 (37.94) & $9.65(4.67)$ & $7.66(2.62)$ \\
\hline \multicolumn{5}{|c|}{ EQ-5D index (maximum 1)‡ } \\
\hline PEPT & $0.53(0.26)$ & $0.63(0.22)$ & $0.77(0.19)$ & $0.76(0.20)$ \\
\hline CONV & $0.47(0.29)$ & $0.64(0.26)$ & $0.67(0.32)$ & $0.74(0.25)$ \\
\hline \multicolumn{5}{|c|}{ EQ-5D VAS (range 0-100)‡ } \\
\hline PEPT & $64.42(15.71)$ & $69.88(18.56)$ & $74.75(16.11)$ & $75.80(14.27)$ \\
\hline CONV & $61.48(17.20)$ & $69.42(21.22)$ & $70.96(20.46)$ & $75.46(14.60)$ \\
\hline
\end{tabular}

Data are based on raw data; mean (SD).

${ }^{*}$ A decrease means improvement.

†Muscle strength $(\%)=$ left-right difference, relative to the non-affected side.

$\ddagger$ An increase means improvement.

§Only for participants with upper extremity affected.

ๆOnly for participants with lower extremity affected.

${ }^{* *}$ Not all participants completed all assessments at all time points.

10MWT, 10-metre walk test; CONV, conventional treatment; DASH, disability of arm, shoulder and hand; EQ-5D, EuroQol-5D; LLTQ, Lower Limb Tasks Questionnaire; PEPT, pain exposure physical therapy; SF-36, Short Form 36; TUG, timed up-and-go test; VAS, visual analogue scale.

\section{Adverse events}

None of the patients reported serious adverse events or disease deterioration. In the CONV group, several patients reported drug-related side effects (nausea, constipation), but these were managed by temporarily reducing the dose or changing medication.

\section{DISCUSSION}

\section{Statement of principal findings}

With the results of this first randomised controlled, single-blinded trial on PEPT, we cannot state that PEPT is superior to CONV for patients with CRPS-1. Patients in both treatment groups experienced a clinically important decrease in ISS-RV, but we did not find a significant between-group difference. We did observe a significantly larger improvement of AROM for patients treated with PEPT. The ITT analysis showed no other differences between treatment groups. The PP analysis showed larger and significant between-group effects on ISS-RV, VAS-pain, AROM, PDI, SF-36, LLTQ and TUG.

\section{Meaning of the study}

Both groups improved substantially on ISS-RV (6.7 points for the PEPT group and 6.2 points for the CONV group), although there was no significant between-group difference. We also observed a relevant improvement in disability for both groups, which we also consider an important outcome measure.

This study indicates that CRPS- 1 may be treated with well-tolerated PEPT, without the use of medication, thus avoiding adverse effects and high costs of medication. However, owing to certain shortcomings in the execution of this trial, as mentioned hereafter in the discussion, while the within-group differences for both treatments suggest improvement, the between-group differences do not provide evidence that PEPT offers additional benefit over CONV. This could be relevant for a cost-effectiveness analysis. Because of the maximum duration of five treatment sessions, PEPT is a low-cost intervention: the number of physical therapy sessions in CONV was much higher than that with PEPT. ${ }^{17}$ The 
Table 5 Estimated differences between treatment groups based on linear mixed models for both intention-to-treat and PP analyses

\begin{tabular}{|c|c|c|c|c|}
\hline Variable & $\begin{array}{l}\text { ITT estimated } \\
\text { difference }(95 \% \mathrm{Cl})\end{array}$ & p Value & $\begin{array}{l}\text { PP estimated } \\
\text { difference }(95 \% \mathrm{Cl})\end{array}$ & p Value \\
\hline ISS-RV & $0.96(-1.56$ to 3.48$)$ & 0.45 & $2.90(0.10$ to 5.70$)$ & 0.04 \\
\hline VAS-pain & $0.61(-0.70$ to 1.92$)$ & 0.36 & $1.61(0.06$ to 3.16$)$ & 0.04 \\
\hline McGill Pain Questionnaire & $0.01(-0.83$ to 0.85$)$ & 0.99 & $0.06(-0.84$ to 0.95$)$ & 0.89 \\
\hline Active range of motion & $0.51(0.07 \text { to } 0.94)^{*}$ & 0.02 & $0.75(0.28$ to 1.23$)$ & 0.003 \\
\hline Skin temperature & $-0.52(-1.53$ to 0.50$)$ & 0.31 & $0.30(-0.72$ to 1.33$)$ & 0.55 \\
\hline Pain Disability Index & $4.98(-1.32$ to 11.28$)$ & 0.12 & 7.91 (1.06 to 14.76$)$ & 0.03 \\
\hline Muscle strength $\dagger$ & $6.42(-4.65$ to 17.48$)$ & 0.25 & $12.64(-0.40$ to 25.68$)$ & 0.06 \\
\hline SF-36 overall mean & $-4.74(-11.85$ to 2.36$)$ & 0.19 & $-6.64(-13.19$ to -0.08$) \ddagger$ & 0.05 \\
\hline DASH & $6.47(-5.97$ to 18.90$)$ & 0.30 & $9.98(-3.29$ to 23.24$)$ & 0.13 \\
\hline LLTQ daily activity & $5.11(-0.45$ to 10.68$)$ & 0.07 & $-22.04(-28.78$ to -15.29$)$ & $<0.001$ \\
\hline 10MWT§ & NA & NA & NA & NA \\
\hline TUG & $1.14(-1.77$ to 4.05$)$ & 0.42 & 11.27 (8.11 to 14.43$)$ & $<0.001$ \\
\hline EQ-5D index & $-0.01(-0.10$ to 0.08$)$ & 0.79 & $-0.06(-0.17$ to 0.06$)$ & 0.33 \\
\hline EQ-5D VAS & $2.41(-4.69$ to 9.50$)$ & 0.50 & $0.45(-7.98$ to 8.89$)$ & 0.91 \\
\hline \multicolumn{5}{|c|}{$\begin{array}{l}\text { Estimated between-group differences, based on linear mixed models with unstructured repeated covariance, where treatment and } \\
\text { measurement in time are factors in the model and outcome at baseline is a covariate. A between-group difference is defined as the score of } \\
\text { the conventional treatment group minus the score of the PEPT group. } \\
\text { *The mean improvement in active range of motion is } 0.51 \text { points greater in the PEPT group than in the conventional treatment group. } \\
\text { †Muscle strength (\%)=left-right difference, relative to the non-affected side. } \\
\text { †The mean improvement in SF-36 is } 6.64 \text { points greater in the PEPT group than in the conventional treatment group. } \\
\text { §Analyses with the 10MWT could not be performed due to lack of power. } \\
\text { 10MWT, 10-metre walk test; DASH, disability of arm, shoulder and hand; EQ-5D, EuroQol-5D; ISS-RV, Impairment level Sum Score-- } \\
\text { Restricted Version; ITT, intention-to-treat; LLTQ, Lower Limb Tasks Questionnaire; PP, per protocol; SF-36, Short Form 36; TUG, timed } \\
\text { up-and-go test; VAS, visual analogue scale. }\end{array}$} \\
\hline
\end{tabular}

outcomes of a cost-effectiveness analysis will be published in a subsequent paper, but based on the previous statements it seems that, from an economic point of view, PEPT could be recommended to patients as well as by caregivers and health insurance companies.

The effects reported in table 5 are estimated effects. We did not include a treatment $\times$ time interaction to the analysis, as this did not contribute to the model. The difference in outcome between treatments was achieved after 3 months (T1) and remained stable over time.

\section{Strengths and limitations of the study}

Our trial included a number of aspects to minimise bias. We prospectively registered the trial and followed the published protocol. ${ }^{17}$ Patients were randomised, allocation was concealed and the assessor was blinded, although we did not systematically record whether patients unblinded the assessor. Small groups of correctly instructed therapists conducted the treatments at different locations of our university hospital. The therapists applying the experimental treatment were specifically trained to perform PEPT. The physical therapists who applied CONV were the same therapists as those

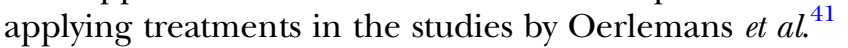

A homogeneous group of patients was included, with baseline characteristics comparable to those of the normal patient population. This argues for generalisability of our findings in the population. However, we only included patients with one affected extremity, so we cannot draw conclusions about the effect of PEPT for patients with more than one affected extremity. Furthermore, the median duration of symptoms was relatively short (6.0 months, IQR 4-9) and only $12.5 \%$ of patients reported at our outpatient clinic $\geq 12$ months after the inciting event. Therefore, we cannot generalise the results of this study to chronic patients.

The inclusion rate was disappointing and the maximum duration of the study forced us to accept a lower number of participants than was calculated for the optimal sample size. No specific measures were undertaken to compensate for non-compliance or loss-to-follow-up and after randomisation, a substantial number of patients $(15 / 56 ; 26.8 \%)$ opted out of their assigned treatment and did not follow the trial protocol. As a result, this trial was underpowered and the primary analysis was based on mixed groups; thus, the actual effect was diluted. These are important limitations of our trial.

We could not foresee so many patients changing treatment group before having finished the initial allocated treatment. Since the initial inclusion rate was low, the funding organisation allowed us to take 1 year extra but without extra financial support. Owing to financial limitations, we could not afford to exceed the extra inclusion period.

Our hospital is known for its research on CRPS-1, and patients referred to our hospital might have had the expectation to receive a new treatment. The larger number of patients that switched to the PEPT group directly after randomisation might be due to this expectation. However, we did not evaluate possible preferences 


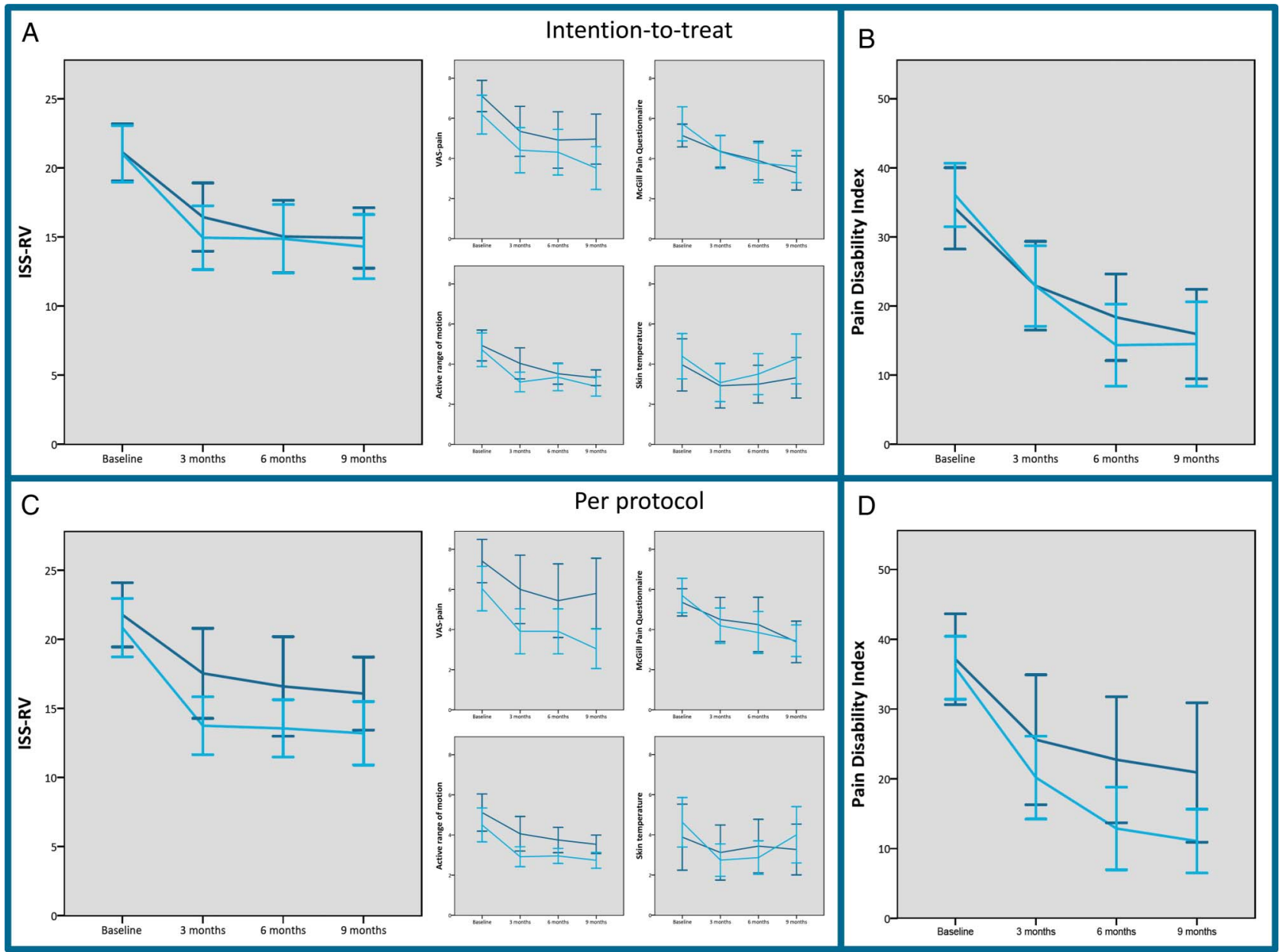

Figure 2 Outcomes of the Impairment level Sum Score-Restricted Version (ISS-RV) and its subscales, and the Pain Disability Index. Data are based on raw data; mean $(95 \% \mathrm{Cl})$. Light lines, pain exposure physical therapy group. Dark lines, conventional treatment group. (A) Primary outcome ISS-RV and its subscales, intention-to-treat. (B) Pain Disability Index, intention-to-treat. (C) Primary outcome ISS-RV and its subscales, per protocol. (D) Pain Disability Index, per protocol (ISS-RV, Impairment level Sum Score-Restricted Version; VAS, visual analogue scale).

prior to randomisation. In future trials, we should explore the opinions and expectations of the patients better prior to inclusion and randomisation. Furthermore, there should be better education about the implication of randomisation in order to minimise switching after inclusion.

A disadvantage of randomised controlled trials is that some patients refrain from participation because of randomisation; they do not want to risk being allocated to the control intervention. Patients who do participate may be disappointed when they are assigned to the control group, which can result in dropouts or patients switching to the experimental group. ${ }^{42}$ One might question whether a conventional randomised controlled trial is the most appropriate form of research in such cases.

After completion of this trial, a Cochrane review was published, showing the lack of evidence of most CONV modalities, ${ }^{7}$ making it difficult to place the results of this trial in perspective. A placebo-controlled trial would reveal the actual treatment effect, without the risk of switching or potentially biasing factors. However, it seems rather undoable to conduct a placebo-controlled physical therapy trial, given the nature of the treatment, the intensity of the condition and the clear request for help in most patients.

A relatively new type of study design, called cohort multiple randomised controlled trial, tackles the aforementioned problems regarding recruitment and allocation and might be a good alternative design in populations like the one under study. ${ }^{43}$

Owing to switching of treatment groups, shifts in baseline characteristics occurred for the PP analysis. The CONV group contained more patients with an affected upper extremity and the dominant side was affected more often in this group. These differences, however, were not significant. According to adjusted analyses, introducing these variables to the model had little influence on the outcome and correction for the variables in the analysis appeared to be unnecessary.

ISS is specifically developed for measuring severity of CRPS-1. A disadvantage of this score is that continuous data are converted to a 1 to 10 scale, which leads to loss 
of data. However, ISS provides a balanced score, in which all domains are represented equally. Since several trials have used ISS as an outcome measure, this allows for comparison of results between trials. In ISS-RV, measurement of limb volume is excluded. This is a rather irreproducible measure with low sensitivity, and causes increased variability in the ISS scores, rendering it less useful. ${ }^{24}$ We expect that excluding measurement of limb volume will not affect the reliability of the sum score.

In the study protocol of this trial, we mentioned three other secondary outcome measures (PAR, the Seven Days Physical Activity Recall questionnaire; IPAQ the International Physical Activity questionnaire; and Personal Activity Monitor, an accelerometer). However, data sets of the outcomes were too incomplete and therefore unsuitable for analysis.

Not all patients who were treated with PEPT adhered to the treatment protocol: 10 patients received some form of medication or other CONV. Most of this treatment was prescribed by their general practitioners, who were not involved in the trial, and in some cases it was not clear whether the treatment was CRPS related.

\section{Strengths and limitations in relation to other studies}

Ek et al studied PEPT in a cohort of 106 adult patients with long-lasting CRPS-1. Function improved in $90 \%$ of patients, and they observed full functional recovery in $46 \%$ of patients. ${ }^{14}$ In 2011, we published an article on the safety of PEPT, reporting that we did not observe exacerbations of CRPS-1 symptoms during intensive stress-loading exercises. A second remarkable finding was that pain relief and complete remission of CRPS-1 symptoms could be reached without specific CRPS-1 medication and/or other invasive treatments. ${ }^{15}$

This study also showed decreased pain intensity, increased AROM and decreased disability in the PEPT group, without disease deterioration.

In another publication, we showed the results of a mediation analysis on pain-related fears (fear-avoidance beliefs, pain catastrophising and kinesiophobia) in the treatment of CRPS-1 with PEPT. We found no indication that pain-related fears mediate the reduction of disability and pain.

\section{Unanswered questions and future research}

In this study, we observed no deterioration shortly after finishing PEPT; however, future studies should explore the long-term tenability of PEPT.

Future research should also focus on the question whether PEPT is applicable to all patients with CRPS-1, both acute and chronic, and whether psychological or cognitive factors or differences in physical characteristics need to be taken into account. Detection of and insights into these factors would help us when making decisions about an appropriate individual intervention.

In future studies, perhaps especially with (chronic) pain patients, researchers should be attentive to expectations of potential participants in order to preclude a large number of participants from switching treatment groups.

\section{CONCLUSION}

We cannot state that PEPT is superior to CONV for patients with CRPS-1. However, patients allocated to PEPT did experience a greater improvement in AROM compared to those allocated to CONV. In the ITT analysis, all other outcomes showed improvement over time, but we were not able to detect significant between-group differences. The improvement in the PEPT group was achieved in a maximum of five treatment sessions and without the use of medication.

Interested readers can find full details of the trial protocol in the Supplemental Appendix, available with the full text of this article, and at http://www. biomedcentral.com/1471-2474/13/58.

\section{Author affiliations}

${ }^{1}$ Radboud Institute for Health Sciences, Radboud University Medical Center, Nijmegen, The Netherlands

${ }^{2}$ Department of Rehabilitation, Radboud University Medical Center, Nijmegen, The Netherlands

${ }^{3}$ Department of Anesthesiology, Pain and Palliative Care, Radboud University Medical Center, Nijmegen, The Netherlands

${ }^{4}$ Department of Orthopedics, Physical Therapy, Radboud University Medical Center, Nijmegen, The Netherlands

${ }^{5}$ Department of Epidemiology, Biostatistics and Medical Technology Assessment, Radboud University Medical Center, Nijmegen, The Netherlands ${ }^{6}$ Department of Medical Psychology, Radboud University Medical Center, Nijmegen, The Netherlands

${ }^{7}$ Department of Surgery, Radboud University Medical Center, Nijmegen, The Netherlands

${ }^{8}$ Research Group Musculoskeletal Rehabilitation, HAN University of Applied Sciences, Nijmegen, The Netherlands

Acknowledgements The authors would like to acknowledge Tjarda Tromp, who performed all of the measurements and data management; Maria Reinders, who performed the randomisation; Rob Oostendorp, who substantially contributed to the conception and design of the study; Anouk Puts and Marijke Tolsma, who carried out the pain exposure physical therapy; and Peter Paul Mazure, Jan Oosterhof and Riet Doppen, who carried out the conventional physical therapy.

Contributors KJB wrote the paper. HvdM, RTMvD, FPK, HS and JPMF contributed to the design of the work. JPMF obtained funding. HvdM and JPMF facilitated recruitment. FPK carried out the pain exposure physical therapy. RTMvD carried out the conventional treatment. HG developed the analyses. MWGN-vdS was the project manager. HvdM, RTMvD, FPK, HG, HS, MWGN-vdS, JPMF and JBS contributed to the drafts.

Funding The Netherlands Organisation for Health Research and Development (ZonMw) financially supported this study (grant number 170991004).

\section{Competing interests None declared.}

Ethics approval The study was approved by the state regional ethical committee and is registered at http://www.clinicaltrials.gov, NCT00817128, and at http://www.trialregister.nl, NTR 2090.

Provenance and peer review Not commissioned; externally peer reviewed.

Data sharing statement Data for the current article, including the primary and secondary outcome measures, are available on request from the corresponding author. Data on additional research, regarding the mediation analysis on the working mechanism of pain exposure physical therapy, are available through https://osf.io/q5js8/. These data include patient characteristics and psychological outcome measures. 
Open Access This is an Open Access article distributed in accordance with the Creative Commons Attribution Non Commercial (CC BY-NC 4.0) license, which permits others to distribute, remix, adapt, build upon this work noncommercially, and license their derivative works on different terms, provided the original work is properly cited and the use is non-commercial. See: http:// creativecommons.org/licenses/by-nc/4.0/

\section{REFERENCES}

1. Bruehl S, Harden RN, Galer BS, et al. External validation of IASP diagnostic criteria for Complex Regional Pain Syndrome and proposed research diagnostic criteria. International Association for the Study of Pain. Pain 1999;81:147-54.

2. Marinus J, Moseley GL, Birklein F, et al. Clinical features and pathophysiology of complex regional pain syndrome. Lancet Neurol 2011;10:637-48.

3. Schwartzman RJ. New treatments for reflex sympathetic dystrophy. N Engl J Med 2000;343:654-6.

4. Maleki J, LeBel AA, Bennett GJ, et al. Patterns of spread in complex regional pain syndrome, type I (reflex sympathetic dystrophy). Pain 2000;88:259-66.

5. Van Rijn MA, Marinus J, Putter $\mathrm{H}$, et al. Spreading of complex regional pain syndrome: not a random process. J Neural Transm 2011;118:1301-9.

6. Dworkin $\mathrm{RH}, \mathrm{O}$ 'Connor $\mathrm{AB}, \mathrm{Kent} \mathrm{J}$, et al. Interventional management of neuropathic pain: NeuPSIG recommendations. Pain 2013;154:2249-61.

7. O'Connell NE, Wand BM, McAuley J, et al. Interventions for treating pain and disability in adults with complex regional pain syndrome. Cochrane Database Syst Rev 2013;4:CD009416.

8. Perez RS, Zollinger PE, Dijkstra PU, et al, CRPS I task force. Evidence based guidelines for complex regional pain syndrome type 1. BMC Neurol 2010;10:20.

9. Vlaeyen JW, Linton SJ. Fear-avoidance and its consequences in chronic musculoskeletal pain: a state of the art. Pain 2000;85:317-32.

10. Keefe FJ, Rumble ME, Scipio CD, et al. Psychological aspects of persistent pain: current state of the science. J Pain 2004;5:195-211.

11. Vlaeyen JWS, Linton SJ. Fear-avoidance model of chronic musculoskeletal pain: 12 years on. Pain 2012;153:1144-7.

12. Taub E, Uswatte G, Mark VW, et al. The learned nonuse phenomenon: implications for rehabilitation. Eura Medicophys 2006;42:241-56.

13. Maihöfner C, Handwerker HO, Neundorfer B, et al. Cortical reorganization during recovery from complex regional pain syndrome. Neurology 2004;63:693-701.

14. Ek JW, van Gijn JC, Samwel H, et al. Pain exposure physical therapy may be a safe and effective treatment for longstanding complex regional pain syndrome type 1: a case series. Clin Rehabil 2009;23:1059-66.

15. Van de Meent H, Oerlemans M, Bruggeman A, et al. Safety of "pain exposure" physical therapy in patients with complex regional pain syndrome type 1. Pain 2011:152:1431-8.

16. Hoffmann TC, Glasziou PP, Boutron I, et al. Better reporting of interventions: template for intervention description and replication (TIDieR) checklist and guide. BMJ 2014;348:g1687.

17. Barnhoorn KJ, Oostendorp RA, van Dongen RT, et al. The effectiveness and cost evaluation of pain exposure physical therapy and conventional therapy in patients with complex regional pain syndrome type 1 . Rationale and design of a randomized controlled trial. BMC Musculoskelet Disord 2012:13:58.

18. Frölke JP, van Rumund $A$, de Waardt $D$, et al. [Complex regional pain syndrome type 1 ? In $77 \%$ of people had a different diagnosis]. Ned Tijdschr Geneeskd 2009;153:550-3.

19. Harden RN, Bruehl S, Stanton-Hicks M, et al. Proposed new diagnostic criteria for complex regional pain syndrome. Pain Med 2007;8:326-31.

20. Jensen MP, Karoly P. Self-report scales and procedures for assessing pain in adults. In: Turk DC, Melzack R, eds. Handbook of pain assessment. 2nd edn. New York, USA: Guilford Press, 2001:15-34.

21. Vanderiet $\mathrm{K}$, Adriaensen $\mathrm{H}$, Carton $\mathrm{H}$, et al. The McGill Pain Questionnaire constructed for the Dutch language (MPQ-DV).
Preliminary data concerning reliability and validity. Pain 1987;30:395-408.

22. Norkin CC, White DJ. Measurement of joint motion. A guide to goniometry. 4th edn. Philadelphia, USA: F.A. Davis Company, 2009.

23. Oerlemans HM, Graff MJ, Dijkstra-Hekkink JB, et al. Reliability and normal values for measuring the skin temperature of the hand with an infrared tympanic thermometer: a pilot study. $J$ Hand Ther 1999;12:284-90.

24. Perez RS, Keijzer C, Bezemer PD, et al. Predictive value of symptom level measurements for complex regional pain syndrome type I. Eur J Pain 2005;9:49-56.

25. Oerlemans HM, Goris RJ, Oostendorp RA. Impairment level sumscore in reflex sympathetic dystrophy of one upper extremity. Arch Phys Med Rehabil 1998;79:979-90.

26. Perez RS, Oerlemans HM, Zuurmond WW, et al. Impairment level SumScore for lower extremity complex regional pain syndrome type I. Disabil Rehabil 2003;25:984-91.

27. Vaneker M, Wilder-Smith $\mathrm{OH}$, Schrombges $\mathrm{P}$, et al. Impairments as measured by ISS do not greatly change between one and eight years after CRPS 1 diagnosis. Eur J Pain 2006;10:639-44.

28. ICF-WHO. International classification of functioning, disability and health. Geneva, Switzerland: World Health Organization, 2001.

29. Soer R, Koke AJ, Vroomen PC, et al. Extensive validation of the Pain Disability Index in three groups of patients with musculoskeletal pain. Spine 2013;38:e562-8.

30. Bohannon RW. Test-retest reliability of the MicroFET 4 hand-grip dynamometer. Physiother Theory Pract 2006;22:219-21.

31. Ware JE Jr, Sherbourne CD. The MOS 36-iten Short-Form Health Survey (SF-36). I. Conceptual Framework and Item Selection. Med Care 1992;30:473-83.

32. Veehof MM, Sleegers EJ, van Veldhoven $\mathrm{NH}$, et al. Psychometric qualities of the Dutch language version of the Disabilities of the Arm, Shoulder, and Hand questionnaire (DASH-DLV). J Hand Ther 2002;15:347-54.

33. McNair PJ, Prapavessis $\mathrm{H}$, Collier $\mathrm{J}$, et al. The lower-limb tasks questionnaire: an assessment of validity, reliability, responsiveness, and minimal important differences. Arch Phys Med Rehabil 2007;88:993-1001.

34. Jackson AB, Carnel CT, Ditunno JF, et al. Gait and Ambulation Subcommittee. Outcome measures for gait and ambulation in the spinal cord injury population. J Spinal Cord Med 2008;31:487-99.

35. Scivoletto $G$, Tamburella $F$, Laurenza $L$, et al. Validity and reliability of the $10-\mathrm{m}$ walk test and the 6-min walk test in spinal cord injury patients. Spinal Cord 2011;49:736-40.

36. Poncumhak $\mathrm{P}$, Saengsuwan J, Kamruecha $\mathrm{W}$, et al. Reliability and validity of three functional tests in ambulatory patients with spinal cord injury. Spinal Cord 2013;51:214-17.

37. Furlan JC, Noonan V, Singh A, et al. Assessment of disability in patients with acute traumatic spinal cord injury: a systematic review of the literature. J Neurotrauma 2011;28:1413-30.

38. Rossier P, Wade DT. Validity and reliability comparison of 4 mobility measures in patients presenting with neurologic impairment. Arch Phys Med Rehabil 2001;82:9-13.

39. Schoppen T, Boonstra A, Groothoff JW, et al. The Timed "up and go" test: reliability and validity in persons with unilateral lower limb amputation. Arch Phys Med Rehabil 1999;80:825-8.

40. Janssen MF, Pickard AS, Golicki D, et al. Measurement properties of the EQ-5D-5L compared to the EQ-5D-3L across eight patient groups: a multi-country study. Qual Life Res 2013;22:1717-27.

41. Oerlemans HM, Oostendorp RA, de Boo T, et al. Adjuvant physical therapy versus occupational therapy in patients with reflex sympathetic dystrophy/complex regional pain syndrome type I. Arch Phys Med Rehabil 2000;81:49-56.

42. Verkooijen HM, Roes K, van Gils $\mathrm{CH}$. Cohort multiple randomized controlled trial: a solution for the evaluation of multiple interventions [article in Dutch]. Ned Tijdschr Geneeskd 2013;157: A5762.

43. Relton C, Torgerson D, O'Cathain A, et al. Rethinking pragmatic randomised controlled trials: introducing the "cohort multiple randomised controlled trial” design. BMJ 2010;340:c1066. 